\title{
C-reactive protein point of care testing in the management of acute respiratory infections in the Vietnamese primary healthcare setting - a cost benefit analysis
}

Yoel Lubell ${ }^{1,2^{*}}$ D, Nga T. T. Do ${ }^{3}$, Kinh V. Nguyen ${ }^{4}$, Ngan T. D. Ta ${ }^{4}$, Ninh T. H. Tran ${ }^{4}$, Hung M. Than ${ }^{4}$, Long B. Hoang ${ }^{3}$, Poojan Shrestha ${ }^{1,5}$, Rogier H. van Doorn ${ }^{1,3}$, Behzad Nadjm ${ }^{1,3}$ and Heiman F. L. Wertheim ${ }^{1,3,6}$

\begin{abstract}
Aim: We assess the cost-benefit implications of C-reactive protein (CRP) testing in reducing antibiotic prescription for acute respiratory infection in Viet Nam by comparing the incremental costs of CRP testing with the economic costs of antimicrobial resistance averted due to lower antibiotic prescribing.

Findings: Patients in the CRP group and the controls incurred similar costs in managing their illness, excluding the costs of the quantitative CRP tests, provided free of charge in the trial context. Assuming a unit cost of $\$ 1$ per test, the incremental cost of CRP testing was $\$ 0.93$ per patient. Based on a previous modelling analysis, the 20 percentage point reduction in prescribing observed in the trial implies a societal benefit of $\$ 0.82$ per patient. With the low levels of adherence to the test results observed in the trial, CRP testing would not be cost-beneficial. The sensitivity analyses showed, however, that with higher adherence to test results their use would be cost-beneficial.
\end{abstract}

Keywords: C- reactive protein, Cost-benefit, Antibiotic, Primary care, Vietnam

\section{Background}

It is estimated that $80-90 \%$ of antibiotic prescription occurs in primary care, of which half are for acute respiratory infection (ARI) [1,2]. Antibiotic use is unrestricted in Viet Nam, and prescription and sales of antibiotics for ARI is very common in- and outside primary health care settings [3] despite a predominantly viral etiology [4]. Approximately $70 \%$ of primary care patients in Viet Nam are prescribed antibiotics, and ARI is the reason for $51 \%$ of these [5]. Treatment decisions are at best based on clinical examination, which in both low and high income settings is of poor accuracy in identifying when antibiotics are required, and is often inadequately performed $[3,6,7]$.

\footnotetext{
* Correspondence: yoel@tropmedres.ac

${ }^{1}$ Centre for Tropical Medicine and Global Health, Nuffield Department of Medicine, University of Oxford, Oxford, UK

${ }^{2}$ Mahidol Oxford Tropical Medicine Research Unit, Faculty of Tropical Medicine, Mahidol University, 420/6 Rajvithi Road, Bangkok 10400, Thailand Full list of author information is available at the end of the article
}

The interaction between antimicrobial consumption and antimicrobial resistance (AMR) is complex, however, it is widely accepted that safe reductions in consumption will have the desirable effect of mitigating the burden of AMR [8]. A recent modelling study estimated the economic costs of AMR per antibiotic consumed, equating with the societal gains for every course of antibiotics averted [9]. For example, in the Thai context, the consumption of a full course of beta-lactams was associated with an economic cost of $\$ 10.8$ due to AMR.

Several biomarker tests have been evaluated for this purpose in the context of ARIs in primary care, and C-reactive protein (CRP) has been shown to have high discriminatory power in distinguishing between viral and bacterial infections, in the range of $85-95 \%$ sensitivity and $50-75 \%$ specificity [10-12]. A meta-analysis of clinical trials concluded that CRP tests can safely reduce antibiotic prescribing [13].

Point-of-care (PoC) CRP tests are commercially available and can be performed in primary care using 
capillary blood samples, with results available within minutes $[14,15]$. This approach is already taken in a number of high income countries such as Norway and Sweden [16] and is recommended by Public Health England and the National Institute for Health and Clinical Excellence (NICE) [17]. In low and middle income countries (LMICs), and in Asia in particular, no such tests are in routine use and antibiotic dispensing is often unregulated or regulation is poorly enforced and resistance levels are high and increasing [18-21]. Despite the urgent need to improve antibiotic targeting in these settings, the necessary evidence on the cost-effectiveness of biomarker testing is scarce.

A randomised control trial in Viet Nam compared CRP PoC testing with routine care in the management of ARIs in primary care, finding significant reductions in antibiotic prescription without compromising patient recovery and satisfaction [22]. To determine whether the incremental cost of introducing CRP tests is economically justifiable, this needs to be compared with the societal costs of AMR the tests could avert. In this short report, we use primary cost data collected in the trial and the output of the modelling analysis of the economic costs of AMR per antibiotic consumed to perform a cost-benefit analysis of CRP testing in primary care in the Vietnamese setting.

\section{Methods}

This cost-benefit analysis of CRP testing takes a societal perspective. Primary cost data were collected in a clinical trial on CRP testing in primary care as described in the Additional file 1: Tables S1 and S2, comparing the costs of managing ARI in the intervention group as compared with controls. The CRP readers and reagents used in the study were donated by the manufacturer, with a purchase cost per kit of approximately $\$ 3$ and for a single reader $\$ 1000$. If implemented at scale, CRP testing could be carried out using simple lateral flow devices that can be used by relatively untrained personnel. Such tests have been shown to be accurate and available at under $\$ 0.5[15,23]$. By way of comparison World Health Organisation (WHO) pre-qualified lateral flow malaria rapid diagnostic tests (RDTs) are available at under $\$ 0.3$. We conservatively assume here a unit cost of $\$ 1$ per test, allowing for added costs for import tariffs, shipment, training, and other peripheral expenses.

The trial results showed no difference in clinical outcomes between the study arms, therefore the benefits considered here relate only to the societal costs of AMR averted due to lower prescribing. Estimates for the economic cost of AMR per antibiotic prescribed are taken from a modelling analysis where these were calculated in the US and Thai contexts [9]. The Thai costs were adjusted by a factor of 0.38 using the ratio of 2017 GDP per capita (PPP) in Viet Nam to that of Thailand $(0.38 *$ $\$ 10.8=\$ 4.1)$. We thus assign an economic cost of AMR of $\$ 4.1$ per full course of broad spectrum beta-lactams, the drug class most often prescribed in the study. The net benefit of CRP testing is calculated as

$$
N M B c r p=\triangle p A B * c A M R-(\triangle D C+C t)
$$

where NMB is the net monetary benefit of CRP testing, $\triangle p A B$ is the percentage difference in prescribing between patients in the CRP group and controls; cAMR is the cost of AMR per antibiotic consumed; $\triangle D C$ is the difference in direct medical care as observed in the trial; and $C t$ is the direct cost of the CRP tests. A positive net monetary benefit indicates that CRP testing is cost-beneficial. All costs are assumed to be incurred at the time patients are presenting at the health centre, therefore no discounting is applied.

A three-way sensitivity analysis was carried out for key drivers of the net-monetary benefit: 1) the cost of the CRP tests, ranging from $\$ 0.5$ to $\$ 3 ; 2$ ) adherence to test results indicating low CRP (i.e. not prescribing antibiotics to patients with low CRP concentrations, which occurred in $64 \%$ of cases in the trial context) ranging from 50 to $100 \%$; and 3) the economic cost of AMR, ranging from $\$ 0$ to $\$ 14$ per course of antibiotics, using the upper bound of the range of costs of AMR per full course of broad spectrum beta-lactam as described in Shrestha et al. (2018) [9], adjusted by the per-capita GDP ratios of Viet Nam and Thailand.

The data were analyzed and the cost-benefit analysis were run in $\mathrm{R}$ version 3.2.2 ( $\mathrm{R}$ Foundation for Statistical Computing, Vienna, Austria) and using the $\mathrm{R}$ Color Brewer package.

\section{Results}

\section{Trial primary outcome and costs}

There was a 20 percentage point risk difference in prescribing on first attendance between patients in the intervention group (43\%) and the controls (63\%). As detailed in the Additional file 1: Table S1, the direct costs of management of ARI in the trial were \$1.24 and \$1.31 in the CRP group and the controls, respectively, with no significant difference $(p=0.28)$. The slight reduction in costs were explained by the lower rate of antibiotic prescribing in the intervention group.

\section{Modelled cost-benefit outcomes}

With the additional assumed cost per CRP test of $\$ 1$, the incremental cost per patient with the use of the test was $\$ 0.93$. With a cost of AMR per full course of antibiotics of $\$ 4.1$, the use of the test results in a negative net-monetary benefit, implying that with a 20 percentage point reduction in prescribing, the use of the test was 

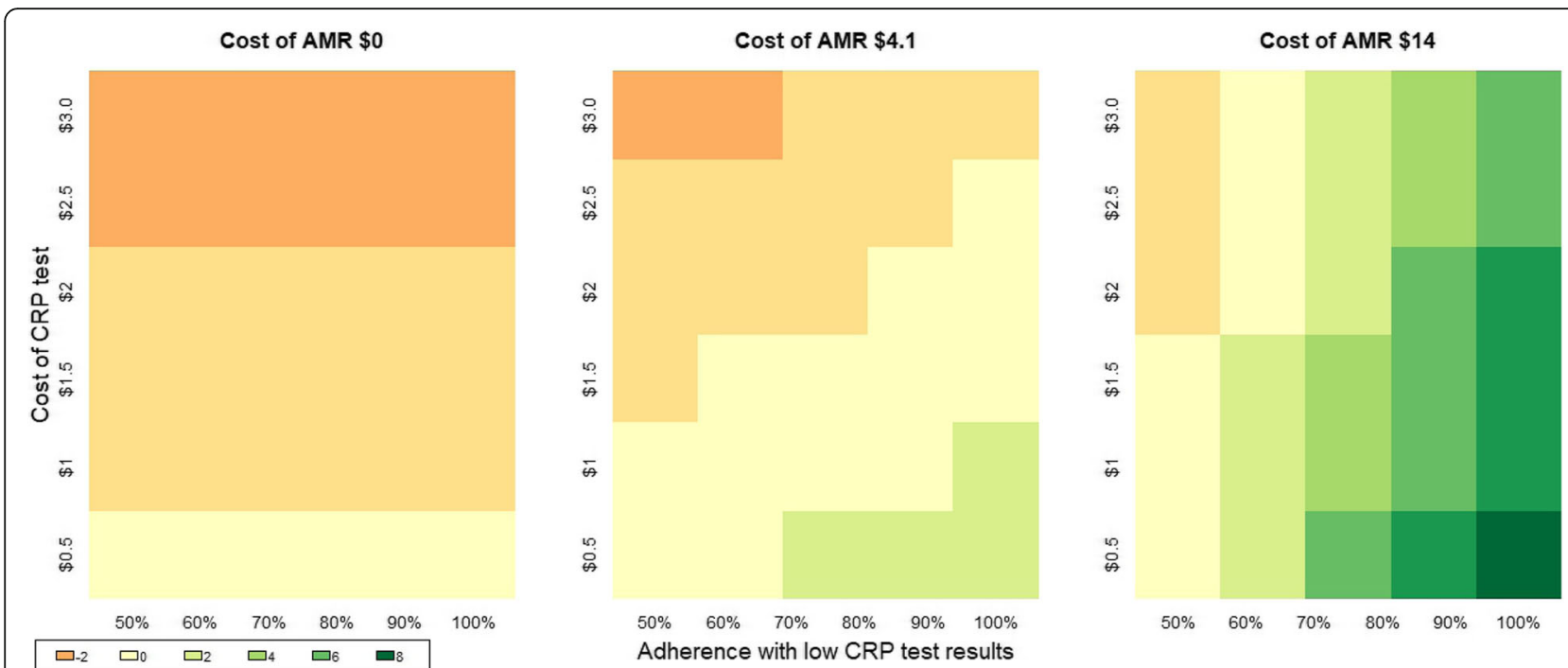

Fig. 1 Net benefit for CRP testing by unit cost, test adherence, and cost of AMR. The 3 panels indicate the net-benefit of CRP testing in response to different configurations of the cost of the CRP test, the degree to which health workers adhere with the test results, and the economic cost of AMR per full course of antibiotic averted. The range of colours reflect the net-benefit of the CRP tests, with dark orange areas indicating instances where the use of the test is not cost-beneficial, and dark green areas where the test is most-cost-beneficial. With the exclusion of the costs of AMR (\$0), a CRP test would be at best cost-neutral if it was low cost. With the inclusion of the costs of AMR, using either the baseline estimate of $\$ 4.1$ or a higher estimate of $\$ 14$, CRP testing would be cost-benficial even if the cost of the test was as high as $\$ 3$, providing adherence with test results was high

not cost-beneficial. Figure 1 presents the sensitivity analysis for the net-benefit of the tests to their unit cost, the adherence to negative test results, and to the economic cost of AMR. This suggests that without consideration of the costs of AMR, the use of the tests is not cost-beneficial even with a low cost test and with high adherence to their results. With the inclusion of a cost of AMR of $\$ 4.1$ per course of antibiotics, the test has a positive net-benefit if adherence exceeds $70 \%$ and the cost of the test is $<=\$ 0.5$, or with adherence at $80 \%$ and a unit cost per test of $\$ 1$. A higher cost of AMR of $\$ 14.1$ per full course of antibiotics would imply a positive $\mathrm{NMB}$ so long as adherence exceeded $60 \%$, even if the cost per test was as high as $\$ 3$.

\section{Discussion}

The trial demonstrated that CRP testing could safely reduce antibiotic prescription in primary health care settings. The primary cost data indicate that other than the cost of the test, there were no added direct medical costs and potential cost savings associated with the intervention. Assuming a unit cost per test of $\$ 1$, the incremental cost per patient would be $\$ 0.93$. With an estimated societal cost of AMR of $\$ 4.1$ per course of a broad spectrum beta-lactam, the use of the tests would not appear to be cost-beneficial due to the limited adherence to their results. The sensitivity analysis, however, indicated that the tests could be cost-beneficial if healthcare workers refrained from prescribing to patients with low CRP at higher rates than observed in the trial.

\section{Implications of the study}

The Wellcome Trust AMR Review estimated that by 2050 global economic losses due to AMR could accumulate to $\$ 124$ trillion [24]. Who should be funding interventions to mitigate the spread of AMR is a challenging question. Patients or even policy makers in LMICs might not view this as a priority over interventions with more tangible short term benefits; this would imply limited and sub-optimal uptake from the global community perspective in its efforts to tackle AMR. The AMR Review appropriately highlighted the need for a global funding mechanism ('Diagnostic Market Stimulus pots'), similar to the Global Fund for AIDS, Tuberculosis and Malaria, dedicated specifically to the development and scale-up of diagnostics and other interventions that safely reduce human antimicrobial consumption. This analysis suggests that investment of these resources in CRP testing in the current context could be cost-beneficial, providing adherence to their results is high.

The vast proportion of antibiotics dispensed for ARI through private pharmacies without prescription in Viet Nam needs to be properly considered [25]. Targeting the private sector could dramatically increase the impact of an intervention such as PoC CRP testing on unnecessary antibiotic use, but has major programmatic challenges. An obvious solution is to enforce the laws that are in 
place since 2006 prohibiting unregulated antibiotic sales, driving these patients to primary and community-care centres where they can be examined and tested. However, as indicated by the trial findings and this cost-benefit analysis, high adherence to the test is critical to ensuring their impact and cost-effectiveness. To achieve this, the introduction of CRP tests could be integrated into a broader public health campaign that includes training and education for both healthcare workers and patients, to maximize the benefits of both interventions and achieve a behavioural shift away from widespread use of antibiotics for minor complaints [26].

\section{Strengths and limitations}

This analysis benefitted from detailed primary costing data from a large clinical trial in Viet Nam evaluating an intervention to address the urgent need for safe reductions in antibiotic prescribing in primary care. To our knowledge these are the only such data from an LMIC setting. Use of the economic costs of AMR averted in a cost-benefit analysis of the intervention also represents an advancement in our ability to perform economic evaluations of such interventions.

The study has numerous limitations. The primary measure of effectiveness in the trial was the proportion of antibiotic prescriptions averted but approximately one third of patients that were not prescribed an antibiotic went on to obtain them elsewhere. If the intervention were to be rolled out in routine care, this should be supplemented by further training and education for healthcare workers and patients to ensure better adherence to the test. Such programmes will incur higher costs, which were not accounted for in the analysis. The sensitivity analysis carried out here indicates that investment in such education and training programmes for patients and healthcare workers to improve adherence to CRP test results could itself be cost-effective.

The CRP test used in the trial relied on a quantitative reader, requiring some degree of technical experience, with a relatively high cost per test, whereas in the model we assume that lower cost lateral flow devices are used instead. It is possible that such devices would have a different impact on prescribing than that observed in the trial.

The methods used to quantify the costs of AMR averted due to reductions in prescribing have numerous limitations detailed in the paper describing their estimation [9]. In addition to these limitations, the adaptation of the costs of AMR calculated in the Thai setting to the Viet Nam context also assumed that Viet Nam and Thailand have similar epidemiological profiles for the prevalence of resistant infections which may not be the case.

As well as guiding whether or not to prescribe an antibiotic, CRP testing might also influence prescribing in terms of choice of antibiotic and duration of treatment, with implications for costs, health outcomes and AMR. As there was no evidence of this occurring in the trial this was not incorporated in the analysis.

Beyond the impact on AMR are other possible costs and health implications associated with CRP testing that were not accounted for. Adverse reactions occur in a small proportion of antibiotic courses, but the frequency of antibiotic use makes them account for approximately a quarter of all adverse events recorded in the hospital setting [27, 28]; a study of adverse drug reactions in emergency department visits found antibiotics to be implicated in a fifth of cases [29]. Second, while over-treatment is a challenge in all income settings, in many LMICs under-treatment of respiratory infection in patients with restricted access to antibiotics imposes a huge morbidity and mortality toll $[30,31]$. In such settings CRP testing could therefore have a direct beneficial impact on health outcomes, through identification of patients that require antibiotic therapy and may not otherwise be detected [32].

\section{Conclusion}

Use of CRP test in the context of primary care in LMICs is likely to incur a modest incremental cost, but this can be offset by the economic costs of AMR averted, providing adherence to their results is high. Whether patients or health providers in LMICs can and should shoulder the tangible costs is contestable; this study suggests that international donor support for these purposes is economically warranted. With low cost tests available and validated, large scale implementation of CRP point of care tests is feasible. Implementation of CRP testing on national scales will pave the way for novel and better systems that are currently cost-prohibitive once these are better validated and available at affordable price ranges.

\section{Additional file}

Additional file 1: Primary costing data from a clinical trial of CRP guided treatment for respiratory illness in vietnamese primary care facilities. (DOCX 297 kb)

\section{Abbreviations}

AMR: Antimicrobial resistance; ARI: Acute respiratory infections; CBA: Costbenefit analysis; CRP: C reactive protein; GDP: Gross Domestic Product; LMIC: Low and middle income country; NHTD: National hospital for tropical diseases; NICE: National Institute for Health and Clinical Excellence; NMB: Net monetary benefit; PoC: Point of care; RDT: Rapid diagnostic test; US: United States; WHO: World Health Organization

\section{Acknowledgements}

We would like to express our sincere thanks to the doctors, nurses and patients from the ten participating healthcare stations for their collaboration during the study implementation. We would also like to thank Alere Technologies, Norway for providing the reagents and equipment for CRP testing. 


\section{Funding}

This work was supported by the Wellcome of Great Britain [089276/Z/09] and the Centre for Disease Dynamics Economics and Policy (CDDEP), Washington DC, USA, as part of the Global Antibiotic Resistance Partnership (GARP).

\section{Availability of data and materials}

The datasets are available from the corresponding author on reasonable request.

\section{Authors' contributions}

$Y L$ and NTTD were responsible for conception and study design. NTTD and LBH led the data collection supervised by KVN and HFLW. NTDT, NTHT, HMT took part in getting ethical approval and training for study sites. YL and PS led the health economic analysis. Manuscript drafting was done by YL, NTTD and HFLW. All authors have contributed to the final editing and approval.

\section{Ethics approval and consent to participate}

The trial was approved by the ethical committees of the National Hospital for Tropical Diseases in Hanoi (39/IRB-NHTD) and the Oxford University Tropical Research Ethics Committee (OxTREC Reference: 176-12). Permission for this study also was obtained from local authorities. Patients who fulfilled the inclusion criteria were asked to participate by signing the consent form. The cost surveys were included in these approvals and consent procedures. The trial is registered at ClinicalTrials.gov under number NCT01918579.

\section{Consent for publication}

Not applicable.

\section{Competing interests}

All authors have no competing interests to declare.

\section{Publisher's Note}

Springer Nature remains neutral with regard to jurisdictional claims in published maps and institutional affiliations.

\section{Author details}

${ }^{1}$ Centre for Tropical Medicine and Global Health, Nuffield Department of Medicine, University of Oxford, Oxford, UK. ${ }^{2}$ Mahidol Oxford Tropical Medicine Research Unit, Faculty of Tropical Medicine, Mahidol University, 420/6 Rajvithi Road, Bangkok 10400, Thailand. ${ }^{3}$ Oxford University Clinical Research Unit, Ha Noi, Viet Nam. ${ }^{4}$ National Hospital for Tropical Diseases, Hanoi, Viet Nam. ${ }^{5}$ Infectious Diseases Data Observatory, University of Oxford, Oxford, UK. ${ }^{6}$ Department of Medical Microbiology, Radboudumc Center of Infectious Diseases, Radboudumc, Nijmegen, Netherlands.

\section{Received: 21 August 2018 Accepted: 26 September 2018}

\section{Published online: 04 October 2018}

\section{References}

1. Goossens H, Ferech M, Vander Stichele R, Elseviers M, Group EP. Outpatient antibiotic use in Europe and association with resistance: a cross-national database study. Lancet. 2005;365(9459):579-87.

2. Shallcross LJ, Davies DSC. Antibiotic overuse: a key driver of antimicrobial resistance. Br J Gen Pract. 2014;64(629):604-5.

3. Larsson M, Falkenberg T, Dardashti A, Ekman T, Tornquist S, Kim Chuc NT, et al. Overprescribing of antibiotics to children in rural Vietnam. Scand J Infect Dis. 2005;37(6-7):442-8.

4. Do AH, van Doorn HR, Nghiem MN, Bryant JE, Hoang TH, Do QH, et al. Viral etiologies of acute respiratory infections among hospitalized Vietnamese children in Ho Chi Minh City, 2004-2008. PLoS One. 2011;6(3):e18176.

5. Chalker J. Improving antibiotic prescribing in Hai Phong Province, Viet Nam: the "antibiotic-dose" indicator. Bull World Health Organ. 2001;79(4):313-20.

6. Arnold SR, To T, Mclsaac WJ, Wang EE. Antibiotic prescribing for upper respiratory tract infection: the importance of diagnostic uncertainty. J Pediatr. 2005;146(2):222-6.

7. Chandler Cl, Nadjm B, Boniface G, Juma K, Reyburn H, Whitty CJ. Assessment of children for acute respiratory infections in hospital outpatients in Tanzania: what drives good practice? Am J Trop Med Hyg 2008;79(6):925-32
8. Holmes AH, Moore LS, Sundsfjord A, Steinbakk M, Regmi S, Karkey A, et al. Understanding the mechanisms and drivers of antimicrobial resistance. Lancet. 2016;387(10014):176-87.

9. Shrestha P, Cooper BS, Coast J, Oppong R, Thuy NDT, Phodha T, et al. Enumerating the economic cost of antimicrobial resistance per antibiotic consumed to inform the evaluation of interventions affecting their use Antimicrob Resist Infect Control. 2018;7(1):98.

10. Lubell Y BS, Dunachie S, Tanganuchitcharnchai A, Watthanaworawit W, Paris D, Mayxay M, Peto TJ, Dondorp A, White NJ, et al. Performance of C-reactive protein and Procalcitonin to distinguish viral from bacterial and malarial causes of fever in Southeast Asia. BMC Infect Dis. In Press.

11. Minnaard MC, van de Pol AC, de Groot JA, De Wit NJ, Hopstaken RM, van Delft $\mathrm{S}$, et al. The added diagnostic value of five different $\mathrm{C}$-reactive protein point-of-care test devices in detecting pneumonia in primary care: a nested case-control study. Scand J Clin Lab Invest. 2015;75(4):291-5.

12. Srugo I, Klein A, Stein M, Golan-Shany O, Kerem N, Chistyakov I, et al. Validation of a novel assay to distinguish bacterial and viral infections. Pediatrics. 2017:e20163453.

13. Aabenhus R, Jensen JU, Jorgensen KJ, Hrobjartsson A, Bjerrum L. Biomarkers as point-of-care tests to guide prescription of antibiotics in patients with acute respiratory infections in primary care. The Cochrane database of systematic reviews. 2014;11:CD010130.

14. Brouwer N, van Pelt J. Validation and evaluation of eight commercially available point of care CRP methods. Clin Chim Acta. 2014.

15. Phommasone K, Althaus T, Souvanthong P, Phakhounthong K, Soyvienvong $L$, Malapheth $\mathrm{P}$, et al. Accuracy of commercially available c-reactive protein rapid tests in the context of undifferentiated fevers in rural Laos. BMC Infect Dis. 2016;16:61.

16. Jakobsen KA, Melbye H, Kelly MJ, Ceynowa C, Molstad S, Hood K, et al. Influence of CRP testing and clinical findings on antibiotic prescribing in adults presenting with acute cough in primary care. Scand J Prim Health Care. 2010;28(4):229-36.

17. Cooke J, Butler C, Hopstaken R, Dryden MS, McNulty C, Hurding S, et al. Narrative review of primary care point-of-care testing (POCT) and antibacterial use in respiratory tract infection (RTI). BMJ open respiratory research. 2015;2(1):e000086.

18. Van Boeckel TP, Gandra S, Ashok A, Caudron Q, Grenfell BT, Levin SA, et al. Global antibiotic consumption 2000 to 2010: an analysis of national pharmaceutical sales data. Lancet Infect Dis. 2014;14(8):742-50.

19. Laxminarayan $R$, Van Boeckel TP. The value of tracking antibiotic consumption. Lancet Infect Dis. 2014;14(5):360-1.

20. Kang $\mathrm{Cl}$, Song JH. Antimicrobial resistance in Asia: current epidemiology and clinical implications. Infection \& chemotherapy. 2013;45(1):22-31.

21. Phu VD, Wertheim HF, Larsson M, Nadjm B, Dinh QD, Nilsson LE, et al. Burden of hospital acquired infections and antimicrobial use in Vietnamese adult intensive care units. PLoS One. 2016;11(1):e0147544.

22. Nga DTT NT, Ninh Tran, Hung TM, Bich VTN, Long HB, et.al. Point-of-care Creactive protein testing to reduce inappropriate use of antibiotics for acute respiratory infections in adults and children in the Vietnamese primary health care setting: a multi-Centre randomised controlled trial. Lancet Glob Health 2016:4(9):e633-ee41.

23. FIND. CRP Landscape. The Foundation for Innovative New Diagnostics. 2017. https://www.finddx.org/wp-content/uploads/2018/03/CRP_List_2018_ 02_26.pdf.

24. O'Neil J. Antimicrobial resistance: tackling a crisis for the health and wealth of nations. 2015

25. Nga do TT, Chuc NT, Hoa NP, Hoa NQ, Nguyen NT, Loan HT, et al. Antibiotic sales in rural and urban pharmacies in northern Vietnam: an observational study. BMC pharmacology \& toxicology. 2014;15:6

26. Rune Aabenhus J-USJ. Biomarker-guided antibiotic use in primary care in resource-constrained environments. Lancet Glob Health. 2016.

27. Stavreva G, DP A, Pandurska R. Marev. Detection of adverse drug reactions to antimicrobial drugs in hospitalized patients. Trakia Journal of Sciences. 2008;6:7-9.

28. Granowitz EVBR. Antibiotic adverse reactions and drug interactions. Crit Care Clin. 2008;24(2):421-42.

29. Shehab N, Patel PR, Srinivasan A, Budnitz DS. Emergency department visits for antibiotic-associated adverse events. Clin Infect Dis. 2008;47(6):735-43.

30. Laxminarayan R, Matsoso P, Pant S, Brower C, Rottingen JA, Klugman K, et al. Access to effective antimicrobials: a worldwide challenge. Lancet. 2015. 
31. Mendelson M, Røttingen J-A, Gopinathan U, Hamer DH, Wertheim H, Basnyat B, et al. Maximising access to achieve appropriate human antimicrobial use in low-income and middle-income countries. Lancet. 2016:387(10014):188-98.

32. Lubell $Y$, Althaus T, Blacksell SD, Paris DH, Mayxay M, Pan-Ngum W, et al. Modelling the impact and cost-effectiveness of biomarker tests as compared with pathogen-specific diagnostics in the Management of Undifferentiated Fever in remote tropical settings. PLoS One. 2016;11(3): e0152420.

Ready to submit your research? Choose BMC and benefit from:

- fast, convenient online submission

- thorough peer review by experienced researchers in your field

- rapid publication on acceptance

- support for research data, including large and complex data types

- gold Open Access which fosters wider collaboration and increased citations

- maximum visibility for your research: over $100 \mathrm{M}$ website views per year

At $B M C$, research is always in progress.

Learn more biomedcentral.com/submissions 